\title{
Interactions fluides-structures en biomécanique : exemple des globules rouges humains
}

\author{
Fluid-structure interactions in Biomechanics : the example \\ of human red blood cells
}

par D. Barthes-Biesel

UMR CNRS 6600, Université de Technologie de Compiègne

Fluid-structure interactions are often met in Biomechanics. Indeed, tissues and blood vessels (arteries, veins) deform easily in order to adapt to the imposed fluid stresses. In the case of microcirculation, the capillary vessels may be considered as rigid. but then the cells (red and white blood cells) have to deform. This paper presents some of the main approaches used when modeling the flow of cells suspended in a viscous liquid. The flow Reynolds number is very small. Although the Stokes equations are linear, the presence of free surfaces that can deform significantly, leads to highly non linear effects.

\section{INTRODUCTION}

La biomécanique a pour objectif la compréhension du fonctionnement mécanique du corps humain afin de faire le diagnostic d'un dysfonctionnement éventuel, de proposer un traitement et d'en évaluer l'efficacité. Pour des raisons évidentes, c'est une science très ancienne en liaison étroite avec la médecine. Une difficulté inhérente aux études en biomécanique est qu'en règle générale, on est confronté à des comportements fortement non linéaires. En effet, la plupart des tissus sont mous, donc très déformables, présentent des rhéologies compliquées non-linéaires et anisotropes, parfois même "actives" comme les muscles. Tous ces facteurs font que la biomécanique est restée assez limitée tant sur le plan expérimental que théorique. Actuellement cette science connaît un essor considérable, favorisé par des possibilités d'investigations expérimentales de plus en plus fines, offertes notamment par l'imagerie biomédicale (scanner ou IRM). En complément, il est maintenant possible d'effectuer des modélisations assez complètes grâce à la puissance de calcul des matériels informatiques disponibles actuellement.

Sur le plan mécanique, on a souvent affaire à des situations où il $\mathrm{y}$ a une forte interaction entre la mécanique des fluides et la mécanique des structures. Le cas le plus connu est sans doute l'écoulement dans les grosses artères où l'écoulement est pulsé et la paroi élastique. En première approximation, on peut négliger les efforts visqueux, et les efforts sur la paroi artérielle se réduisent donc principalement aux variations de pression dues aux pulsations cardiaques. Les déformations correspondantes de la paroi artérielle sont assez petites pour que l'on puisse leur appliquer une théorie localement linéaire. On obtient ainsi une évaluation de la vitesse de propagation de l'onde cardiaque le long d'une artère (de l'ordre de $10 \mathrm{~m} / \mathrm{s}$ dans l'aorte). Un autre cas classique d'interactions fluides-structures concerne les écoulements dans des conduites très déformables qui ont la propriété de s'effondrer par flambement lorsque la pression transmurale est négative (la pression transmurale est égale à la différence entre la pression interne et la pression extérieure). Tel est le cas des veines sous l'effet de variations de pression hydrostatique interne dues à un changement de posture. On observe également ces phénomènes dans les voies aériennes supérieures (bronches, trachée, larynx) et dans la microcirculation pulmonaire. Ces vaisseaux sont dits collabables. L'écoulement dans des conduits collabables est très complexe, car il dépend à la fois de la différence de pression motrice aux bornes du vaisseau et de la valeur locale de la pression transmurale qui détermine la forme de la section. Les expériences, comme les modèles numériques, mettent en évidence des modes d'écoulement instables avec des oscillations entretenues où la conduite se ferme et s'ouvre périodiquement. Ce phénomène est notamment à l'origine du ronflement lors du sommeil.

A l'échelle cellulaire, on rencontre des interactions fluidesstructures originales pour lesquelles le nombre de Reynolds est négligeable du fait de la petite taille des cellules (de l'ordre de quelques microns). L'exemple le plus frappant est celui des globules rouges qui se déforment considérablement sous l'effet des contraintes visqueuses imposées par la circulation du sang. A titre d'exemple, ce cas va être développé en détail dans la suite.

Pour en savoir plus, on peut consulter l'ouvrage Biomécanique des Fluides et des Tissus [1]. 


\section{II — PHYSIOLOGIE DU SANG ET DU GLOBULE ROUGE HUMAIN}

\subsection{Le sang}

Le sang humain est une suspension concentrée (concentration volumique de l'ordre de $45 \%$ ) de diverses cellules (globules rouges ou hématies, globules blancs et plaquettes) dans du plasma. Les globules rouges (GR) ont une concentration normale d'environ $5 \times 10^{6}$ cellules $/ \mathrm{mm}^{3}$ et représentent $99 \%$ du volume cellulaire total. Leur agrégation, leur dissociation et leur déformation sous cisaillement jouent donc un rôle essentiel dans le comportement rhéologique du sang entier. Les globules blancs (GB) ont une concentration de l'ordre de 7000 cellules $/ \mathrm{mm}^{3}$. Ils sont plus gros que les GR, mais sur le plan mécanique, ils ne sont à prendre en compte que lors de la circulation dans des microvaisseaux capillaires de diamètre inférieur à $10 \mu \mathrm{m}$. Dans cette situation, le temps de transit des GB sera beaucoup plus élevé que celui des GR. Les plaquettes ont un effet mécanique négligeable lors de l'écoulement du sang, mais jouent un rôle essentiel pour la coagulation. Du fait de sa constitution, le sang a des propriétés non-newtoniennes qui dépendent de la micromécanique de l'écoulement à l'échelle cellulaire. Par conséquent, l'étude de la rhéologie du sang ne sera pas la même selon que l'on s'intéresse à l'écoulement dans les grands vaisseaux ou dans les conduits capillaires. Le milieu de suspension, le plasma, est lui-même une solution complexe de minéraux et de macromolécules. Aux cisaillements modérés, on peut le traiter comme un fluide newtonien dont la viscosité est environ $1,2 \mathrm{mPa} . \mathrm{s}$ à $37^{\circ} \mathrm{C}$.

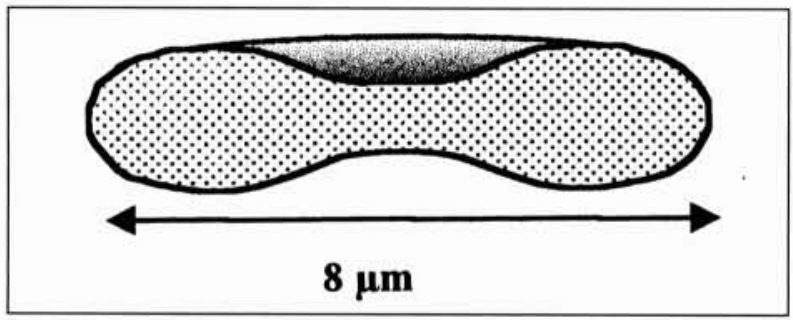

1. Coupe d'un globule rouge normal.

\subsection{Les globules rouges}

Dans leur état normal, les GR sont des disques biconcaves de $8 \mu \mathrm{m}$ de diamètre, remplis d'une solution d'hémoglobine et entourés par une membrane mince (Figure 1). Ces cellules se déforment facilement, ce qui a deux conséquences importantes. Au niveau de la grande circulation et malgré la forte concentration volumique en cellules, la déformation des GR conduit à une faible viscosité apparente du sang (de l'ordre de $5 \mathrm{mPa}$.s pour des taux de cisaillement supérieurs à $100 \mathrm{~s}^{-1}$ ). Cela diminue donc l'énergie que doit fournir le cœur pour pomper le sang dans tout le corps. De plus, au niveau de la microcirculation, les GR arrivent à se faufiler dans des vaisseaux capillaires d'un diamètre bien inférieur au leur. Les globules prennent alors une forme en "parachute" ou en "pantoufle". Ce processus est très important puisque c'est au niveau de la microcirculation que se font les échanges gazeux entre le sang et les tissus.

En conclusion la "déformabilité" des GR est une propriété essentielle, mais qu'il est difficile de quantifier avec préci- sion. En effet, elle résulte de plusieurs propriétés physiques intrinsèques des GR : la viscosité de la solution interne d'hémoglobine, la rhéologie de la membrane et la géométrie discoïdale flasque. La géométrie peut être connue avec précision à partir d'observations microscopiques. La solution interne a un comportement newtonien avec une viscosité qui dépend de la concentration en hémoglobine. A l'état normal, cette viscosité est de l'ordre de $6 \mathrm{mPa} . \mathrm{s}$ à $37^{\circ} \mathrm{C}$. La membrane est constituée d'une mince bicouche lipidique doublée sur sa face interne d'un réseau de protéines. Son épaisseur est de l'ordre de $10^{-8} \mathrm{~m}$, ce qui permet de traiter la membrane comme un milieu continu bidimensionnel. Du fait de cette composition, la membrane du GR a un comportement élastique particulier : sa résistance à la flexion est faible, son module de cisaillement surfacique $\mathrm{G}_{\mathrm{s}}$ est faible également $\left(\mathrm{G}_{\mathrm{s}} \approx 4\right.$ à $\left.5 \cdot 10^{-6} \mathrm{~N} \mathrm{~m}^{-1}\right)$ mais son module de dilatation $\mathrm{A}_{\mathrm{s}}$ est comparativement très élevé $\left(A_{\mathrm{s}} / \mathrm{G}_{\mathrm{s}} \approx 10^{5}\right)$. Ces propriétés impliquent que la membrane se déforme facilement sous cisaillement mais garde sa surface locale constante pendant le processus.

\section{MODÉLISATION DU MOUVEMENT D'UNE CELLULE DANS UN ÉCOU- LEMENT}

On modélise le GR par une capsule constituée d'une membrane (M) fermée, infiniment mince, de module élastique surfacique $E_{s}$, et contenant un liquide interne de viscosité $\lambda \mu$ (domaine fluide $\Omega^{*}$ ). La géométrie au repos de la capsule est supposée connue et sa dimension caractéristique est dénotée $\mathrm{A}$ (par exemple le rayon de la sphère de même volume). Suivant les propriétés qu'on lui donnera, la capsule représentera un globule rouge ou une capsule artificielle. La capsule est suspendue librement dans un liquide visqueux incompressible et newtonien de viscosité $\mu$. On néglige les effets gravitationnels et l'on se place dans un référentiel lié au centre de masse de la particule (Figure 2). Le liquide de suspension est soumis loin de la capsule à un écoulement connu de champ de vitesse $\mathbf{u}_{\infty}$ de module caractéristique U. L'écoulement extérieur du fluide de suspension crée un champ de contraintes visqueuses o qui s'exerce sur la membrane. De même, l'écoulement du liquide intérieur crée un autre champ de contraintes visqueuses $\sigma^{*}$ qui s'exerce aussi sur la membrane. La membrane se déforme donc sous l'action conjuguée de ces deux champs, qui dépendent, bien entendu, de la géométrie déformée de la capsule. On a donc affaire à un problème où la mécanique des fluides et la mécanique des membranes élastiques minces sont étroitement couplées. L'équation de la forme de la capsule est inconnue et doit être déterminée lors de la résolution du problème.

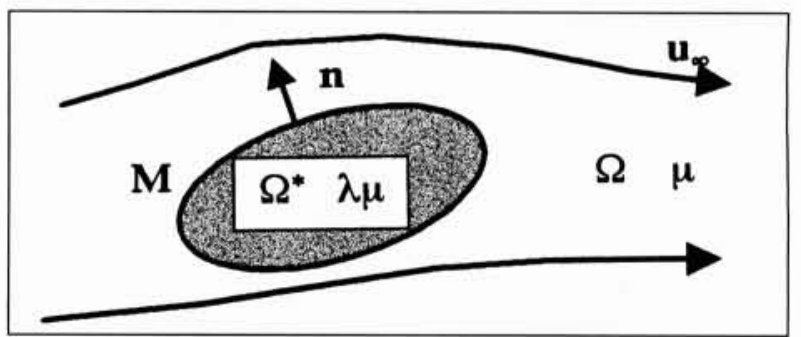

2. Schéma d'une capsule suspendue librement dans un écoulement. 


\subsection{Mouvement des fluides interne et externe}

On suppose que le nombre de Reynolds de l'écoulement autour de la capsule est très petit :

$\operatorname{Re}=\rho \mathrm{UA} / \mu, \operatorname{Re}<<1$.

Cette hypothèse est souvent justifiée par la petite taille des cellules. Les mouvements des fluides interne et externe sont alors décrits par les équations de Stokes, où $\mathbf{u}$ et p sont les champs de vitesse et pression. Les variables relatives à l'écoulement à l'intérieur de la capsule sont affublées d'un astérisque. On a donc :

$$
\begin{array}{ll}
\nabla \cdot \mathbf{u}=0, & \nabla \cdot \mathrm{p}=\boldsymbol{\mu} \nabla^{2} \mathbf{u}, \quad \text { pour } \quad \mathrm{x} \in \Omega \\
\nabla \cdot \mathbf{u}^{*}=0, & \nabla \cdot \mathrm{p}^{*}=\boldsymbol{\lambda} \boldsymbol{\mu} \nabla^{2} \mathbf{u}^{*}, \quad \text { pour } \quad \mathrm{x} \in \Omega^{*} .
\end{array}
$$

Les conditions aux limites associées sont :

- pas de perturbation de l'écoulement loin de la capsule :

$$
\mathbf{u} \rightarrow \mathbf{u}_{\infty} \quad \text { lorsque } \quad|\mathbf{x}| \rightarrow \infty
$$

- non-glissement des fluides au niveau de la membrane et imperméabilité de celle-ci :

$$
\mathbf{u}^{*}(\mathrm{x})=\mathbf{u}(\mathbf{x})=\partial \mathbf{x} / \partial \mathrm{t} \quad \text { pour } \mathbf{x} \in \mathrm{M},
$$

où la vitesse $\partial \mathbf{x} / \partial \mathrm{t}$ d'un point de la membrane est la dérivée temporelle de sa position actuelle $\mathbf{x}$ exprimée en fonction d'une variable lagrangienne qui identifie le point matériel.

- équilibre dynamique de la membrane :

$$
\left[\boldsymbol{\sigma}-\boldsymbol{\sigma}^{*}\right]_{\mathrm{M}} \cdot \mathbf{n}=\varepsilon \mathbf{q} \text {, }
$$

où $\left[\boldsymbol{\sigma}-\boldsymbol{\sigma}^{*}\right]_{\mathrm{M}}$ représente le saut de contraintes visqueuses à travers la membrane, $\mathbf{n}$ le vecteur unitaire normal dirigé vers le fluide externe et $\mathbf{q}$ le chargement exercé par les contraintes visqueuses sur la membrane. Pour fermer le problème, il faut relier $\mathbf{q}$ à la mécanique de la membrane.

\subsection{Mécanique de la membrane}

La membrane étant supposée infiniment mince, les contraintes s'exerçant dans celle-ci peuvent être moyennées à travers l'épaisseur et remplacées par des tensions T. Ces tensions sont reliées au chargement $\mathbf{q}$ par les équations d'équilibre des coques minces [2] :

$$
\mathbf{q}+\nabla_{\mathrm{s}} \cdot \mathbf{T}=0
$$

où $\nabla_{\mathrm{s}}$ représente un gradient pris le long de la surface de la coque. Les composantes du tenseur des tensions de Cauchy $\mathbf{T}$ sont ensuite reliées aux déformations par la loi de comportement de la membrane. On suppose la membrane isotrope dans son plan. Les directions principales de déformation et de tension sont donc confondues. On peut postuler plusieurs lois de comportement différentes. Ces lois sont exprimées dans le repère des directions principales (indices 1 et 2 ) en fonction des élongations principales $\lambda_{1}$ et $\lambda_{2}$. Seule une composante de tension est donnée, l'autre s'obtient en échangeant les rôles des indices 1 et 2 . Le module $E_{\mathrm{s}}$ représente un module de Young surfacique. La relation entre $\mathrm{E}_{\mathrm{s}}$ et le module de cisaillement surfacique $\mathrm{G}_{\mathrm{s}}$ est précisée.

\section{Membrane de type élastomère}

On traite la membrane comme une couche infiniment mince d'un élastomère incompressible en volume et modélisé par une loi de type Mooney-Rivlin :

$T_{11}=\frac{E_{s}}{3 \lambda_{1} \lambda_{2}}\left\{\Psi\left[\lambda_{1}^{2}-\frac{1}{\left(\lambda_{1} \lambda_{2}\right)^{2}}\right]+\Psi\left[\left(\lambda_{1} \lambda_{2}\right)^{2}-\frac{1}{\lambda_{1}^{2}}\right]\right\}$,

$\mathrm{G}_{\mathrm{s}}=\mathrm{E}_{\mathrm{s}} / 3$.

Les coefficients $\Psi$ et $\Psi^{\prime}$ sont sans dimensions et tels que
$\Psi+\Psi^{\prime}=1$. Si on accepte que $\Psi$ et $\Psi^{\prime}$ dépendent des invariants de la déformation, on peut modéliser des comportements complexes de type rigidifiant (membrane de nylon par exemple) ou écrouissant (membrane de gélatine).

\section{Membrane du globule rouge}

Skalak et al. [3] ont proposé de modéliser la membrane du GR par une loi qui comprend deux termes correspondant respectivement aux déformations de cisaillement (mesurées par $\mathrm{E}_{\mathrm{s}}$ ) et aux dilatations de surface (mesurées par $\mathrm{A}_{\mathrm{s}}$ )

$$
\begin{aligned}
& \mathrm{T}_{\mathrm{II}}=\mathrm{E}_{\mathrm{s}} \frac{\lambda_{1}}{4 \lambda_{2}}\left(\lambda_{1}^{2}-1\right)+\mathrm{A}_{\mathrm{s}} \lambda_{1} \lambda_{2}\left[\left(\lambda_{1} \lambda_{2}\right)^{2}-1\right], \\
& \mathrm{G}_{\mathrm{s}}=\mathrm{E}_{\mathrm{s}} / 4 .
\end{aligned}
$$

L'analyse dimensionnelle du problème indique que la solution dépend de plusieurs nombres sans dimensions :

- $\lambda$, le rapport des viscosités interne et externe ;

- $\varepsilon=\mu \mathrm{U} / \mathrm{E}_{\mathrm{s}}$, le rapport entre les forces visqueuses et les forces élastiques ;

- $E_{s} / A_{s}$, et tout autre nombre mesurant les propriétés de la membrane et/ou la géométrie initiale.

\subsection{Solutions de perturbation : capsules sphériques}

L'ensemble des équations (1) à (7) ou (8) constitue un système non linéaire difficile à résoudre dans le cas général. On peut en trouver une solution analytique par la méthode des perturbations régulières si la capsule est initialement sphérique et soumise à de petites déformations. En effet, BarthesBiesel \& Rallison [2] prédisent le mouvement transitoire d'une capsule sphérique à membrane élastique dont le comportement est donné par (7) ou par (8). Ils prédisent également la viscosité apparente d'une suspension diluée de capsules identiques. La déformation d'une capsule à membrane viscoélastique (de viscosité de surface $\mu_{\mathrm{s}}$ ) a été calculée pour un écoulement de cisaillement simple de taux G [4]. Dans ce cas, le modèle prédit que la déformation $\mathrm{D}$ de la capsule augmente avec le taux de cisaillement $G$, jusqu'à une valeur maximum $\mathrm{D}_{\infty} \approx 5 \mu \mathrm{A} / 2 \mu_{\mathrm{s}}$. Aux faibles cisaillements, la déformation est une fonction linéaire de $\mathrm{G}: \mathrm{D} \approx 25 \mu \mathrm{GA} / 4 \mathrm{E}_{\mathrm{s}}$. Simultanément, l'angle d'orientation de la capsule avec les lignes de courant diminue de $45^{\circ}$ à $0^{\circ}$ quand $\mathrm{G}$ augmente.

Une géométrie sphérique n'est évidemment pas adaptée pour représenter un GR humain, mais correspond bien à la plupart des capsules artificielles couramment utilisées en pharmacologie. Toutefois, ce modèle permet de bien comprendre la physique des phénomènes mis en jeu. Enfin, ses prédictions sont en accord qualitatif avec ce qui est observé expérimentalement pour des GR et en accord quantitatif avec ce qui est mesuré pour des capsules artificielles. En effet, Chang \& Olbricht [5] ont mesuré la déformation de capsules artificielles entourées d'une interface de nylon dans différents champs de cisaillement linéaire. Ils utilisent ce modèle pour en déduire le module d'élasticité de l'interface de nylon.

\section{MODÉLISATIONS NUMÉRIQUES}

\subsection{Formulation intégrale des équations de Stokes}

Pour pouvoir aborder le cas de capsules non sphériques soumises à de grandes déformations, il faut avoir recours à 
une solution numérique des équations (1) à (6), accompagnées d'une loi de comportement. Dans de nombreuses applications, on n'est pas intéressé par les détails de l'écoulement dans tout le domaine $\Omega$ ou même à l'intérieur $\Omega^{*}$ de la capsule. On cherche simplement à connaître le mouvement de la capsule ainsi que sa forme et son orientation. Dans ce cas, la formulation intégrale des équations de Stokes est tout à fait appropriée, puisqu'elle relie le champ de vitesse sur les frontières du domaine $\Omega$ aux efforts exercés sur ces frontières. Ces dernières sont constituées de la limite extérieure $\partial \Omega$ de $\Omega$ et de $\mathrm{M}$, la membrane de la capsule. Le principe du calcul consiste à partir d'un état connu de la capsule (généralement on injecte la capsule non déformée en un point de $\Omega$ ) et de suivre sa réponse transitoire dans l'écoulement. Cette méthode a été utilisée pour calculer la déformation d'une capsule dans un écoulement d'élongation pure [6], [7], dans un écoulement de cisaillement simple [8] ou dans un pore hyperbolique [9]. A titre d'exemple, nous allons examiner en détail le modèle de l'écoulement d'une cellule dans un pore cylindrique [10]. Cette configuration correspond à ce qui se passe dans la microcirculation ou dans un pore de filtration.

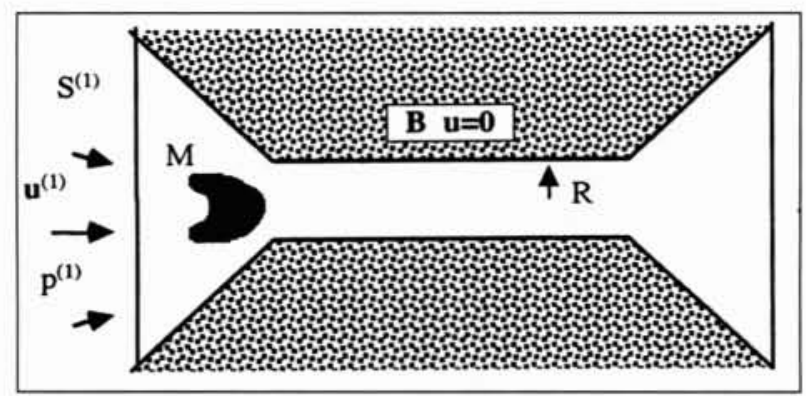

3. Schéma de l'écoulement dans un pore cylindrique.

\subsection{Mouvement d'une cellule dans un pore cylindrique}

L'objectif est de modéliser le mouvement d'une cellule dans un pore de rayon $\mathrm{R}$, plus petit ou du même ordre que la dimension caractéristique A (Figure 3). On impose le débit volumique $\mathrm{Q}$ dans le pore. Le domaine $\Omega$ est délimité par $\mathrm{M}$, la paroi B et par deux sections d'entrée $\mathrm{S}^{(1)}$ et de sortie $\mathbf{S}^{(2)}$, situées loin de la cellule et où donc les vitesses $\mathbf{u}^{(1)}(\mathbf{x})$ et $\mathbf{u}^{(2)}(\mathbf{x})$ sont données (vitesse de l'écoulement sans particule) et où les pressions $\mathrm{p}^{(1)}(\mathbf{x})$ et $\mathrm{p}^{(2)}(\mathbf{x})$ sont uniformes. Le problème fluide à résoudre est décrit par (1) à (5), complétées par les conditions suivantes :

$$
\mathbf{u}(\mathbf{x})=\mathbf{0}, \quad \mathbf{x} \in \mathrm{B},
$$

$$
\mathbf{u}(\mathbf{x})=\mathbf{u}^{(i)}(\mathbf{x}) \text {, et } p(\mathbf{x})=p^{(i)}, \mathbf{x} \in \mathrm{S}^{(i)} ; i=1,2 .
$$

Les pressions $\mathrm{p}^{(1)}$ et $\mathrm{p}^{(2)}$ sont inconnues et doivent être calculées :

$$
\mathrm{p}^{(1)}-\mathrm{p}^{(2)}=\Delta \mathrm{P}_{0}+\Delta \mathrm{P}^{+}(\mathrm{t})
$$

où $\Delta \mathrm{P}_{0}$ est la perte de charge entre $\mathrm{S}^{(1)}$ et $\mathrm{S}^{(2)}$ en l'absence de cellule et où $\Delta \mathrm{P}^{+}(\mathrm{t})$ est la perte de charge additionnelle due à la particule. Le nombre capillaire est alors redéfini comme $\varepsilon=\mu \mathrm{Q} / \pi \mathrm{R}^{2} \mathrm{E}_{\mathrm{s}}$.

Pour des raisons d'économie de temps de calcul, on se limite à des situations à symétrie de révolution où le pore et la cellule ont le même axe de révolution. A l'état stationnaire dans le pore, du fait de la symétrie, le liquide intérieur à la cellule est au repos. Le rapport de viscosité $\lambda$ n'intervient donc pas et on peut lui donner n'importe quelle valeur. On choisit $\lambda=1$, ce qui simplifie notablement l'équation intégrale (bien entendu, le régime transitoire pendant lequel la cellule entre dans le pore est influencé par ce choix de $\lambda$ !). Pour une capsule donnée, le modèle permet de déterminer l'évolution temporelle, lors du passage, de différents paramètres comme la perte de charge additionnelle, la vitesse du centre de masse de la capsule, sa forme, les tensions et les déformations dans la membrane, etc. Il permet aussi d'obtenir les valeurs stationnaires de ces paramètres.

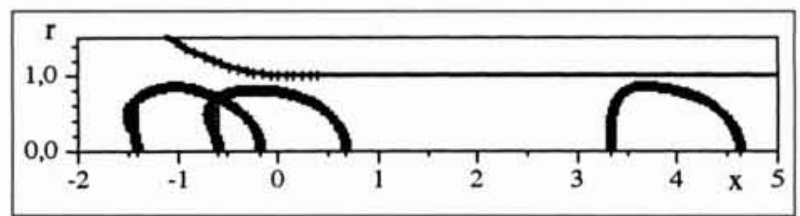

4. Demi-profils successifs d'une capsule de type globule rouge $(\varepsilon=0,02)$. D'après $[10]$.

Afin d'évaluer le rôle de la rhéologie de la membrane, on compare les pertes de charge stationnaires dues à des capsules ellipsoïdales de même géométrie initiale, de rayon $R_{c}$ et de diamètre axial $2 \mathrm{R}_{\mathrm{a}}$. La capsule $\mathrm{C}_{\mathrm{GR}}$ est entourée d'une membrane de type globule rouge, incompressible en surface (loi de comportement (8)) et la capsule $\mathrm{C}_{\mathrm{MR}}$ est entourée d'une membrane de type élastomère (loi de comportement (7)). Une différence importante est que les capsules $C_{M R}$ atteignent une forme stationnaire seulement pour des valeurs du paramètre $\varepsilon$ inférieures à une valeur critique $\varepsilon_{\mathrm{c}}$. Pour $\varepsilon>\varepsilon_{\mathrm{c}}$, la capsule s'étire indéfiniment et va donc se rompre. Un comportement analogue a déjà été prédit pour un écoulement d'élongation pure [6]. Par contre, les capsules $\mathrm{C}_{\mathrm{GR}}$ ont une déformation limitée par la contrainte d'incompressibilité de surface et atteignent un profil limite indépendant de la force de l'écoulement. Une augmentation de $\varepsilon$ résulte seulement en une augmentation des tensions dans la membrane, ce qui peut évidemment conduire à un processus d'éclatement également, mais pour des raisons différentes du cas précédent.

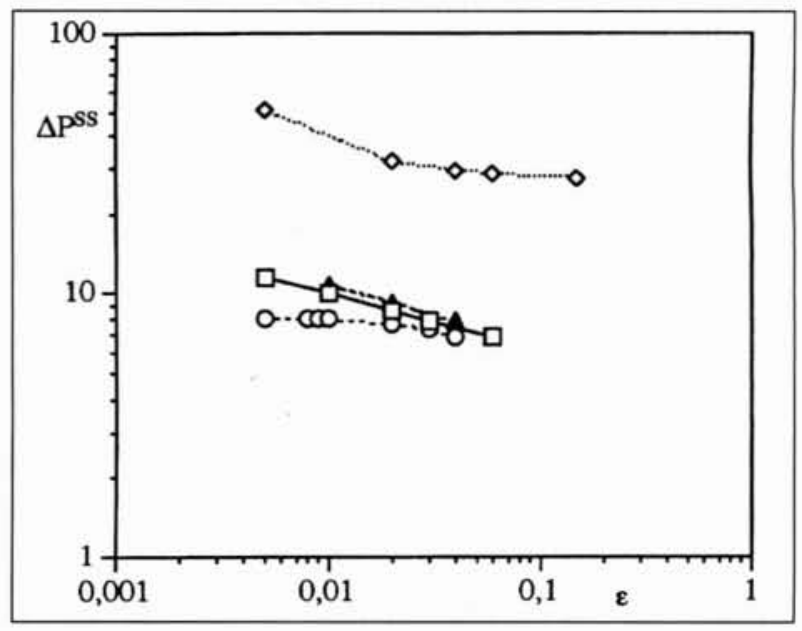

5. Perte de charge stationnaire pour différentes particules. $\square C_{G R}\left(R_{c} / R=0,9, R_{a} / R=0,6\right) ; \diamond: C_{M R}\left(R_{c} / R=0,9\right.$, $\mathbf{R}_{\mathrm{a}} / \mathbf{R}=\mathbf{0 , 6 ) ;} O^{\circ}: \mathbf{C}_{\mathrm{MR}}$ sphérique $\mathrm{A} / \mathbf{R}=\mathbf{0 , 8} ; \boldsymbol{\Delta}: \mathbf{C}_{\mathrm{GR}}$ $\left(R_{c} / R=1,2, R_{a} / R=0,8\right)$. D'après [10]. 
Pour des capsules plus petites que le tube $\left(R_{c} / R=0,9\right.$, $\mathrm{R}_{\mathrm{a}} / \mathrm{R}=0,6$ ), la forme stationnaire est en parachute, comme ce qui est observé lors de la microcirculation (Figure 4). Les pertes de charge stationnaires sont comparées pour des capsules ellipsoïdales ou sphériques isovolumiques ayant une membrane incompressible ou une membrane de type élastomère (Figure 5). Dans tous les cas, la perte de charge stationnaire $\Delta \mathrm{P}^{\mathrm{ss}}$ est faible, dépend peu de $\varepsilon$ (et donc de l'élasticité de la membrane) ou de la géométrie initiale. De plus, la perte de charge stationnaire est peu sensible au comportement mécanique de la membrane (Figure 5).

Pour des capsules plus grosses que le tube $\left(R_{c} / R=1,2\right.$, $R_{a} / R=0,8$ ), la forme stationnaire est en manchon (Figure 6) et est analogue à celle qui est observée pour des globules blancs dans la microcirculation. La capsule $\mathrm{C}_{\mathrm{MR}}$ a tendance à éclater pour $\varepsilon>610^{-3}$. La capsule $\mathrm{C}_{\mathrm{GR}}$ présente une forme et une perte de charge invariante pour $\varepsilon>210^{-2}$ (Figure 5).

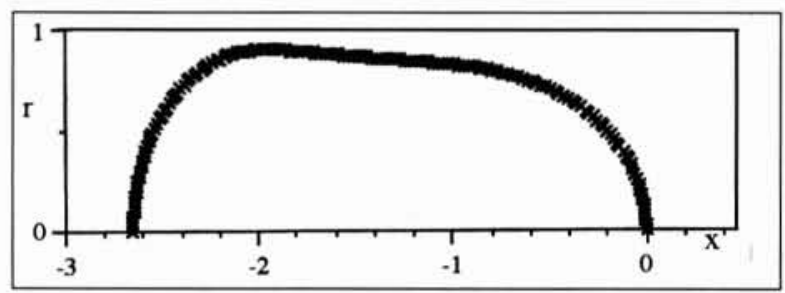

6. Demi-profils d'une cellule $C_{G R}\left(R_{c} / R=1,2\right.$, $\left.R_{\mathrm{a}} / \mathrm{R}=\mathbf{0 , 8}\right), \varepsilon=0,15$. D'après [10]

La conclusion de cette étude numérique est qu'il est pratiquement impossible d'utiliser des expériences de filtration pour évaluer les propriétés mécaniques de la membrane d'une capsule. En effet, ou la particule est plus petite que le pore et la perte de charge additionnelle est si faible qu'elle est difficile à mesurer avec précision. D'autre part, il n'existe pratiquement pas de différence entre différents comportements de la membrane. Si par contre, la capsule est grosse par rapport au tube, alors il faut travailler dans une gamme de valeurs de $\varepsilon$ qui n'est pas commode expérimentalement (débit faible, temps de transit long et risque de colmatage du pore). De plus l'influence de la mécanique de la membrane mesurée par $\varepsilon$ est très faible. De fait, le paramètre le plus sensible est lié à la grosseur relative de la cellule et à son rapport surface/volume. En effet, plus la cellule sera "flasque", plus elle se déformera facilement avec un faible coût énergétique. C'est notamment le cas des globules rouges humains, dont la très grande déformabilité est sans doute une de leurs caractéristiques mécaniques les plus remarquables.

\section{$\mathrm{V} \square$ CONCLUSION}

Il existe des problèmes d'interactions fluides-structures très intéressants aux faibles nombres de Reynolds. Bien que la mécanique des fluides soit linéaire, les problèmes rencontrés sont fortement non linéaires car ils impliquent de grandes déformations de frontières libres. La technique des intégrales de surface se prête bien à la résolution de ces problèmes. Elle est efficace et permet de diminuer la dimension du problème d'un facteur 1. Cette approche est d'ailleurs couramment utilisée en microhydrodynamique : écoulement autour de surfaces irrégulières, écoulement autour de gouttes avec convection d'agents tensio-actifs, etc.

\section{RÉFÉRENCES}

[1] Jafrin (M.-J.) \& Goubel. (F.), Biomécanique des Fluides et des Tissus. Masson ed. (1998).

[2] Barthes-Biesel (D.) \& Rallison (J. M.), J. Fluid Mech. 113, 25 (1981).

[3] Skalak (R.), Tozeren (A.), Zarda (R.-P.), Chien (S.) Biophys. J.. 13. 245 (1973).

[4] Barthes-Biesel (D.) \& Sgaier (H.), J. Fluid Mech. 160, 119 (1985).

[5] Chang (K.-S.) \& Olbricht (W.-L.), J. Fluid Mech, 250, 587 (1993).

[6] Li X. (Z.), BARThes-Biesel. (D.) \& Helmy (A.), J. Fluid Mech. 187, 179 (1988).

[7] PozRidikis (C.), J. Fluid Mech. 216, 231 (1990).

[8] Ramanujan (S.) \& Pozrikidis (C.), J. Fluid Mech. 361. 117 (1998).

[9] Leyrat-Maurin (A.) \& Barthes-Biesel (D.), J. Fluid Mech. 279, 135 (1994).

[10] Quéguiner (C.) \& Barthes-Biesel (D.), J. Fluid Mech. 348, 349 (1997). 